\title{
El análisis de la resiliencia en personas que constituyen parejas mixtas en Andalucía
}

\author{
Analysis of Resilience in Members of Mixed Couples in Andalusia \\ Mercedes GoNZÁLEZ VÉLEZ \\ Universidad de Huelva \\ mercedes@uhu.es \\ Octavio VÁzQUEZ AgUAdo \\ Universidad de Huelva \\ octavio@uhu.es \\ Pablo ÁlVARez PÉrez \\ Universidad de Huelva \\ pablo.alvarez@dstso.uhu.es
}

Recibido: 04/03/2013

Revisado: 18/04/2013

Aceptado: 11/06/2013

Disponible on line: 20/12/2013

\section{Resumen}

Antecedentes. El estudio de la resiliencia está orientado hacia el análisis de los recursos y las respuestas que los sistemas formales e informales activan para prevenir o disminuir los efectos de los problemas sociales. Los matrimonios y parejas mixtas se conforman en un contexto de dificultad por la crisis económica, las diferencias culturales y los recelos grupales hacia los extranjeros. Objetivo. Conocer el grado de resiliencia de los componentes de los matrimonios y parejas mixtas, estableciendo sus principales características resilientes y el/los perfil/es de las personas con alto nivel de resiliencia emparejadas de manera intercultural. Método. Utilizamos la escala de resiliencia de Wagnild y Young (1993) en la versión adaptada por Rodríguez, Pereyra, Gil, Jofré, De Bortoli y Labiano, (2009). Resultados. Los datos obtenidos nos ponen de manifiesto correlaciones significativas entre las variables origen y sexo con la resiliencia, así como la importancia que tienen en la construcción de la resiliencia los aspectos relacionados con la competencia personal y la autoeficacia. Conclusión. Entendemos que es necesario profundizar en los contenidos y los procesos generadores de resiliencia en las relaciones interculturales de estas parejas, como instrumento de aprendizaje extrapolable a marcos profesionales diversos que tengan como objetivos construir escenarios educativos de transformación social, de mejora de la convivencia y de una vida más diversa y rica.

Palabras clave: resiliencia, parejas mixtas; inmigración; relaciones interculturales; competencias personales.

\begin{abstract}
Background. The study of resilience focuses on the analysis of resources and answers activated by formal and informal systems to prevent or lessen the effects of social problems. Married and mixed couples are in a difficult situation due to the economic crisis, to cultural differences and to the existing prejudices towards people from foreign countries. Objective. The aim of this study is to determine the level of resilience in members of interracial marriages and couples. Also, identify the degree of resilience and main characteristics and profiles of people with high levels of resilience in intercultural couples. Method. To complete this study, we have used the resilience scale by Wagnild and Young (1993), adapted by Rodríguez, Pereyra, Gil, Jofré, De Bortoli y Labiano, (2009). Results. The results show significant correlations between the variables of origin and gender and resilience. Aspects related to personal competence and autoefficacy appear to have influence on resilience. Conclusions. We understand the need to further deepen the study of contents and processes causing resilience in the intercultural relationships of these couples in order to create learning instruments able to be extrapolated to different professional areas, with the goal of building educational environments that contribute to social change, to promote coexistence and enriched diversity.
\end{abstract}

Keywords: Resilience, Mixed Couples, Immigration, Intercultural Relations, Personal Competences.

Referencia normalizada: González Vélez, M., Vázquez Aguado, O., y Álvarez Pérez, P. (2013): «El análisis de la resiliencia en personas que constituyen parejas mixtas en Andalucía». Cuadernos de Trabajo Social, 26(2): $275-284$.

Sumario: Introducción. 1. Método: 2. Resultados. 3. Discusión y conclusiones. 4. Referencias bibliográficas. 


\section{Introducción}

Durante décadas, la tendencia en las ciencias sociales ha puesto la atención en los estados patológicos, en los factores de riesgo, y los estudios se han orientado a describir las enfermedades, las causas que pueden explicarlas y sus características o consecuencias. Sin embargo en la actualidad, podemos constatar que se está produciendo un cambio de paradigma: de aquellos orientados a la evaluación, análisis y tratamiento de los problemas sociales a aquellos orientados a los recursos y respuestas que los sistemas formales e informales activan para prevenir o disminuir los efectos de dichos problemas sociales. Integrada en esta visión surge el concepto de resiliencia, cuya interdisciplinariedad permite el tratamiento holístico de áreas de investigación que exploran aspectos personales e interpersonales, y las fuerzas internas que se pueden desplegar para aprender y crecer a través de las situaciones de adversidad (Villalba, 2006).

El término resiliencia ha ido adquiriendo importancia en las ciencias sociales y se ha incorporado, adecuado y precisado su alcance cada vez más (Kotliarenco y Dueñas, 1994; Klotiarenco, Cáceres y Álvarez 1996; Klotiarenco, Cáceres y Fonteccilla, 1997; Barranco, 2009; Rojas, 2010; Muñoz-Silva, 2012), a pesar de haber pasado por un proceso de desarrollo lleno de dificultades y ser variados los enfoques desde los que se la ha investigado (Vanistendael, 1996 y Villalba, 2003, 2004 y 2006; Florentino, 2008; Palma, 2010).

En este proceso se ha pasado de poner el acento en las características individuales y cualidades que presentan las personas resilientes, a definir la resiliencia como el resultado de un proceso de interacción entre el sujeto y su contexto, apareciendo tres aspectos novedosos (Infante, 2002): el concepto de adversidad a la que se enfrenta la persona resiliente, que se entiende como riesgo, trauma o amenaza para su vida y su desarrollo, y que es necesario identificar para ver hasta qué punto le afecta a la persona. El concepto de adaptación positiva se analiza como la superación de la adversidad, se considera positiva cuando la persona alcanza un nivel de desarrollo y de realización tal que quienes están a su alrededor lo perciben como normal o satisfactorio; o cuando no hay manifestación de desajuste emocional o físico, pese a haber vivido una experiencia de adversidad, y cuyo concepto puede variar de una cultura a otra o incluso entre grupos familiares. Por último, el concepto de proceso que tiene en consideración la interacción entre los aspectos familiares, biológicos, afectivos, de historia de vida, sociales, económi$\cos$ y culturales que influyen en el desarrollo y realización integral de la persona.

La resiliencia la entendemos como un proceso, un continuo devenir. No es tanto la persona la que es resiliente como la evolución y la articulación de la misma en su vida. Nunca es absoluta o total, sino que está siempre en construcción. Es una capacidad que resulta dinámica, interactiva y evolutiva. Podemos ser resilientes ante un acontecimiento y no serlo ante otro; ser resiliente con algunas personas y no con otras, en determinados contextos y no en otros (Cyrulnik, 2008). La resiliencia refleja la habilidad de los sistemas para mantener un equilibrio desde que aparecen los acontecimientos estresantes hasta que se superan. De este modo, podemos afirmar que en la concepción de la resiliencia interactúan tres conjuntos de elementos (Garmezy, 1991; Masten y Coastworth, 1998). Aspectos personales: inteligencia, factores cognitivos, sociabilidad, expresividad, autoestima positiva, tener un locus de control interno (Luttar, 1991; Wernery Smith, 1992). Entorno familiar: seguridad en la casa y el entorno, afecto, cercanía y presencia de un adulto que cuida, etc. Apoyo social o externo: constituido por las redes a las que se pertenecen: familia extensa, escuela, vecindad, asociaciones, etc.

$\mathrm{Si}$ el concepto de resiliencia nos aproxima a las capacidades que tienen las personas para hacer frente a los desafíos y problemas a los que se enfrentan, el fenómeno de los matrimonios o parejas mixtas nos remite a uno de los elementos más novedosos en la cambiante sociedad española de principios del siglo XXI. Hay un aumento de parejas o matrimonios mixtos y de familias multiculturales (TognettiBordogna, 1996; Borobio 2003; Giménez, 2003; Esteve y Cortina, 2009; Autora, 2012). Estas uniones constituyen uno de los ámbitos más importantes en los procesos de interculturalidad, sobre todo en lo referido al ámbito cotidiano, así nos lo indica Rodríguez García (2004) al afirmar que «el contexto y formación de uniones mixtas constituyen un espacio de hibridez sociocultural especialmente activo y 
complejo; y que estas dinámicas implican procesos de negociación y acomodación de bagajes socioculturales diversos» (p. 114).

Podemos definir la pareja o el matrimonio mixto como aquellas uniones formadas por personas que proceden de diferentes grupos lingüísticos, religiosos, étnicos o nacionales; esto es, aquellas uniones que incluyen todas las complejidades de lo que podría significar una cultura diferente, teniendo en cuenta que las diferencias culturales son menores o mayores según la procedencia de los componentes de la pareja (Alaminos, 2008). Su significación social procede de conectar mundos diferentes, poner de manifiesto que la comunicación intima en diversidad es posible, nos hace más ricos al aumenta nuestros repertorios cognitivos, emocionales y conductuales, y por último - como nos indica Rodríguez Marcos (2006) - porque son escuelas de la interculturalidad en lo cotidiano.

Este tipo de uniones dan lugar a un espacio complejo donde tienen lugar negociaciones y adaptaciones, donde se realizan elecciones de manera voluntaria, y donde se generan creatividades además de, por supuesto, donde se afrontan conflictos (Albert y Massanet, 2008). En ellos también van disminuyendo las distinciones culturales de las futuras generaciones, al tener la posibilidad de identificarse con más de un grupo de pertenencia. Y por último, la relación de la pareja puede debilitar las actitudes negativas, prejuicios y estereotipos hacia otros grupos (Kalmijn, 1998).

A esta complejidad debemos unir el contexto de crisis en el que se insertan y la alarma social ante las migraciones. El éxito o fracaso de estas parejas y familias interculturales está influenciado por la capacidad de relativizar y superar los prejuicios, la estigmatización social y transformar, al menos en el entorno próximo, las representaciones sociales negativas y los estereotipos asociados al inmigrante (Rodríguez Marcos, 2006). Sólo la percepción positiva mutua (contexto y pareja) garantiza la convivencia intercultural en cualquier ámbito de la vida. Por estas razones señalamos, como objetivos de este trabajo, conocer el grado de resiliencia de los componentes de las parejas mixtas, estableciendo sus principales características resilientes y el perfil o los perfiles de las personas con alto nivel de resiliencia emparejadas de manera intercultural.

\section{Método}

\subsection{Instrumento}

El instrumento empleado ha sido la escala de resiliencia de Wagnild y Young (1993) en la versión adaptada por Rodríguez, Pereyra, Gil, Jofre, De Bortoli y Labiano (2009). Nos animó a usar este instrumento sus propiedades psicométricas, avaladas por la alta fiabilidad obtenida en las investigaciones en las que se ha sido usada con anterioridad (Novella, 2002; Heilemann, Lee y Kury, 2003; Pesce, Assis, Avanci, Santos, Malaquías y Carvalhaes, 2005; Jarami1lo, Ospina, Cabarcas y Humphreys, 2005; Lundman, Strandberg, Eisemman, Gustafson y Brulin, 2007).

Esta escala de resiliencia consta de tres factores: competencia personal y autoeficacia. Autoconfianza y sentido de la vida y evitación cognitiva. Se ha utilizado la escala Likert de 5 puntos. Para medir su fiabilidad hemos empleado el Alpha de Cronbach, que ha aportado un coeficiente de 0.78 . Un análisis más detallado nos pone de manifiesto que en 20 de los ítems la correlación con la escala de resiliencia oscilaba entre 0.37 y 0.65 y es altamente significativa $(p<0,001)$. Para el ítem S es 0.20 , significativa $(p<0,01)$. No alcanza significación estadística en cuatro ítems ( $\mathrm{L}, \mathrm{U}, \mathrm{V}$ y $\mathrm{W}$ ), en los que el coeficiente de correlación es inferior a $0,1,1$ legando a ser negativo en L $(-0,03)$. Sólo dos ítems ( $\mathrm{L}$ y U), presentan un coeficiente de homogeneidad corregido negativo y que son los ítems cuya eliminación permitiría incrementar el valor de Alpha a 0,80 .

Dado que acordamos estudiar el grado de resiliencia aportado por la escala de resiliencia respecto a otras variables, también analizamos el coeficiente Alpha de Cronbach, con objeto de establecer también la fiabilidad. Estratificamos la población de acuerdo con las variables clave, como son sexo y origen. Los resultados aportados son similares al segmentar los datos según origen nacional (Alpha $=0,76)$ o extranjero (Alpha $=0,79)$, situación que también ocurre al estudiar hombres $($ Alpha $=0,77)$ y mujeres de modo separado $($ Alpha $=0,79)$.

\subsection{Participantes}

Han participado un total de 178 personas, de las cuales 84 españolas y 94 extranjeras. Dentro del colectivo nacional, el 61,9 por ciento 
eran varones, mientras que son mayoritarias las mujeres $(62,4$ por ciento) entre las personas de origen extranjero.

En cuanto a la edad, un 46,7 por ciento de los participantes tienen entre 25 y 34 años y un 32,2 por ciento entre 35 y 44 años. Quienes tienen entre 45 y 55 años conforman el 9,1 por ciento del total, mientras que los más jóvenes, entre 18 y 24 años, son el 8,5 por ciento y los mayores de 55 años el 3 por ciento.

La mayoría de las parejas son heterosexuales (193 personas) y sólo 6 personas pertenecen a uniones homosexuales ( 2 de ellas mujeres y 4 hombres). No están casadas el 39,7 por ciento y, cuando lo hacen, la mayoría ha realizado un matrimonio civil (37,2 por ciento). Son muy pocas las registradas como parejas de hecho (1,5 por ciento). El 54 por ciento de las parejas ha convivido 3 años o menos con anterioridad a la formalización de la unión, y el 10 por ciento no ha convivido de forma previa. El 82,4 por ciento estaba soltero antes de iniciar esta relación. El grueso de los participantes $(64,8$ por ciento) no tiene hijos y el 28,1 por ciento sólo tiene uno. En cuanto a las hijas, encontramos que el 71, 4 por ciento no las tiene $\mathrm{y}$ que el 18,6 por ciento tiene una.

Los países de nacimiento de los miembros de la pareja de origen extranjero se sitúan mayoritariamente en Centroamérica y Sudamérica $(42,4$ por ciento), acumulando Colombia el 12,1 por ciento del total. Le siguen los nacidos en algún país europeo ( 32,8 por ciento) y en el continente africano, donde los de origen marroquí representan el 10,6 por ciento de las personas entrevistadas. Los extranjeros nacidos en España representan el 8,6 por ciento del total.

En torno a un tercio de los encuestados (30,2 por ciento), habían permanecido entre uno a tres años en el país antes de unirse a su pareja, el 15,6 por ciento había residido entre 3 y 6 años, el 15,1 por ciento menos de un año, el 9 por ciento ninguno, el 8,5 por ciento más de 10 años y el 6,5 por ciento entre 6 y 10 años.

En relación al lugar en que se conocieron, el 71,4 por ciento conoció a su pareja en España: a través de amistades (37,7 por ciento); en el trabajo (20,6 por ciento) o de otra forma (13,6 por ciento); por internet $(11,6$ por ciento); en vacaciones ( 8,5 por ciento); y por familiares ( 3 por ciento). Por último, el tiempo me- dio de convivencia en más del 50 por ciento de estas parejas oscila entre 8 meses y 6 años.

\section{Resultados}

\subsection{Niveles de resiliencia}

Para obtener la puntuación en la escala de resiliencia se sumaron los valores obtenidos en los 25 ítems, alcanzando una media de 95.51 puntos. Para contextualizar este dato hemos indicado que, para la escala de cinco valores, se considera que el grado de resiliencia es bajo si el total no supera los 86 puntos, moderado si está entre 87 y 104 puntos y alto a partir de 105 puntos. Las dos terceras partes de la población encuestada $(68,5$ por ciento) se encuentra en la franja intermedia de la escala (grado moderado de resiliencia). El tercio restante se divide entre un 14,6 por ciento que muestra una resiliencia baja y un 16,9 por ciento que configura el colectivo con resiliencia alta. Estos niveles han sido construidos a partir de la puntuación media obtenida por las distintas variables, tal y como se muestra en la Tabla 1.

\subsection{Nivel de resiliencia según sexo y edad}

Los hombres presentan un nivel de resiliencia alto similar, ya sean de origen nacional o extranjero $(11,5$ por ciento y 11,4 por ciento, respectivamente), siendo la media de la escala para los hombres autóctonos de 96.00 puntos y para los originarios de otros países de 93.06 puntos. Esta similitud entre los hombres no se da en las mujeres, de manera que sólo el 3,1 por ciento de las mujeres de origen nacional se pueden considerar que tienen alta resiliencia, frente al 32,8 por ciento entre las extranjeras, lo que se traduce en una diferencia superior a las seis unidades en las respectivas medias (92.06 y 98.36 puntos, respectivamente). Atendiendo a estos datos podemos afirmar que más que incidir el origen (media de 94.50 para nacionales y de 96.40 para extranjeros) o el sexo (medias de 94.82 y 96.12 para hombres y mujeres, respectivamente), es la combinación de ambas variables lo que muestra una relación más fuerte con la resiliencia $(p<0,01)$. Esta característica combinada (origen y sexo) es una de las más discriminantes de cuantas se han estudiado. Por lo tanto, se ha decidido analizar los niveles de resiliencia según las variables sociodemográficas analizadas en el cues- 


\begin{tabular}{|c|c|c|c|c|c|c|c|}
\hline \multirow{2}{*}{ Ítem } & \multicolumn{5}{|c|}{ Categorías } & \multirow{2}{*}{ Media } & \multirow{2}{*}{ D.T. } \\
\hline & 1 & 2 & 3 & 4 & 5 & & \\
\hline $\begin{array}{l}\text { A. Generalmente puede percibir una situación desde dife- } \\
\text { rentes perspectivas o puntos de vista (19) }\end{array}$ & 0,6 & 3,9 & 14,0 & 47,8 & 33,7 & 4,10 & 0,82 \\
\hline $\begin{array}{l}\text { B. La confianza en sí mismo/a le permite superar los mo- } \\
\text { mentos y etapas difíciles (17) }\end{array}$ & 1,1 & 5,1 & 14,0 & 46,6 & 33,1 & 4,06 & 0,88 \\
\hline $\begin{array}{l}\text { C. Generalmente maneja los problemas de diversos mo- } \\
\text { dos (2) }\end{array}$ & 0,6 & 5,1 & 21,9 & 49,4 & 23,0 & 3,89 & 0,83 \\
\hline D. Siente que puede realizar diferentes cosas a la vez (9) & 1,1 & 5,6 & 16,9 & 43,8 & 32,6 & 4,01 & 0,91 \\
\hline $\begin{array}{l}\text { E. Cuando está en una situación difícil, generalmente en- } \\
\text { cuentra una salida (23) }\end{array}$ & 0,6 & 3,9 & 14,6 & 56,7 & 24,2 & 4,00 & 0,77 \\
\hline $\begin{array}{l}\text { F. Es capaz de hacer las cosas por sí mismo/a sin depen- } \\
\text { der de los demás (3) }\end{array}$ & 0,6 & 3,4 & 21,3 & 39,9 & 34,8 & 4,05 & 0,87 \\
\hline G. Generalmente encuentra cosas de las que reírse (16) & 0,6 & 3,4 & 11,8 & 38,8 & 45,5 & 4,25 & 0,84 \\
\hline $\begin{array}{l}\text { H. En una emergencia, es alguien en quien las personas pue- } \\
\text { den confiar (18) }\end{array}$ & 0,6 & 0,6 & 8,4 & 46,1 & 44,4 & 4,33 & 0,70 \\
\hline I. Siente orgullo de haber logrado cosas en la vida (6) & 0,0 & 1,1 & 13,5 & 36,5 & 48,9 & 4,33 & 0,75 \\
\hline $\begin{array}{l}\text { J. Ha podido superar situaciones difíciles, porque ha ex- } \\
\text { perimentado dificultades antes (13) }\end{array}$ & 0,6 & 3,4 & 14,0 & 37,6 & 44,4 & 4,22 & 0,85 \\
\hline K. Normalmente, se basta a sí mismo/a (5) & 2,8 & 11,8 & 24,7 & 39,9 & 20,8 & 3,64 & 1,03 \\
\hline L. A veces usted hace cosas por obligación (20) & 2,2 & 8,4 & 18,0 & 47,8 & 23,6 & 3,82 & 0,96 \\
\hline M. Mantiene interés por las cosas (15) & 0,0 & 0,6 & 12,4 & 50,0 & 37,1 & 4,24 & 0,68 \\
\hline N. Su vida tiene sentido (21) & 0,6 & 0,6 & 7,9 & 32,6 & 58,4 & 4,48 & 0,72 \\
\hline $\begin{array}{l}\text { O. Generalmente tiene energía para hacer aquello que tiene } \\
\text { que hacer (24) }\end{array}$ & 1,1 & 1,7 & 16,9 & 47,2 & 33,1 & 4,10 & 0,81 \\
\hline P. Es autodisciplinado/a (14) & 1,1 & 10,1 & 18,5 & 43,8 & 26,4 & 3,84 & 0,97 \\
\hline $\begin{array}{l}\text { Q. Mantiene el interés en aquellas cosas importantes para } \\
\text { usted (4) }\end{array}$ & 0,0 & 0,6 & 6,2 & 47,8 & 45,5 & 4,38 & 0,63 \\
\hline R. Cuando hace planes los lleva a cabo hasta el final (1) & 1,1 & 3,9 & 25,8 & 40,4 & 28,7 & 3,92 & 0,90 \\
\hline S. Acostumbra a tomar las cosas sin preocuparse mucho (7) & 12,4 & 27,0 & 29,8 & 23,0 & 7,9 & 2,87 & 1,14 \\
\hline T. Es amigo de sí mismo (8) & 0,6 & 6,2 & 24,2 & 37,1 & 32,0 & 3,94 & 0,93 \\
\hline $\begin{array}{l}\text { U. Realiza las cosas de una en una (hace una sola cosa a la } \\
\text { vez) (12) }\end{array}$ & 11,8 & 25,8 & 29,8 & 23,0 & 9,6 & 2,93 & 1,16 \\
\hline V. Rara vez se pregunta sobre el objetivo de las cosas (11) & 25,8 & 28,1 & 30,3 & 10,1 & 5,6 & 2,42 & 1,14 \\
\hline W. No insiste en cosas en las que no puede hacer nada (22) & 11,8 & 27,0 & 32,0 & 21,3 & 7,9 & 2,87 & 1,12 \\
\hline $\begin{array}{l}\text { X. Se siente cómodo/a aunque haya gente que no le agra- } \\
\text { da (25) }\end{array}$ & 11,2 & 26,4 & 30,3 & 22,5 & 9,6 & 2,93 & 1,15 \\
\hline Y. Es determinado/a (10) & 0,0 & 0,0 & 21,3 & 66,9 & 11,8 & 3,90 & 0,57 \\
\hline
\end{tabular}

Tabla 1. Análisis descriptivo de la Escala de Resiliencia (ER)

Fuente: Elaboración propia en el marco del Proyecto AFINTA.

Nota: Entre paréntesis se indica el número del ítem de la escala original. 
tionario, no sólo para la población global sino segmentada según el origen, el sexo y la combinación de ambas variables.

Respecto de la edad, podemos afirmar que las personas con más de 44 años tienen un mayor nivel de resiliencia, ya que el 23,8 por ciento de este grupo presenta un nivel alto, si bien es un dato no significativo. Esta tendencia general se reproduce al analizar la información según origen y sexo de modo independiente, observando cómo es relevante entre los hombres: el 25 por ciento presenta un nivel alto. Sin alcanzar la significación, es destacable cómo en los colectivos menos resilientes (mujeres nacionales y hombres extranjeros) la situación se invierte y las pocas personas con un nivel alto de resiliencia no pasan de 34 años.

En cuanto a la edad de la pareja, también se observa mayor resiliencia en las personas cuya pareja tiene más de 34 años, siendo significativa la diferencia de la puntuación media de la escala de resiliencia que oscila desde 90.06 para los menores de 25 años hasta 98.58 para las que tiene más de 44 años $\left(p_{2}<0,01\right)$. Al desglosar según el origen, este incremento con la edad se observa de manera más acusada entre las personas extranjeras, siendo estadísticamente relevante $\left(p_{2}<0,05\right)$ el intervalo que va desde 89.30 puntos (menores de 25 años) a 100.31 (mayores de 44 años). Al igual que se indicó al analizar la edad de la persona encuestada en los colectivos menos resilientes (mujeres nacionales y hombres extranjeros) se observa cómo la edad de las pocas personas con un nivel alto de resiliencia no pasa de 34 años.

\subsection{Estado civil y resiliencia}

Si bien debe decirse que el 84,2 por ciento de las personas encuestadas eran solteras antes de iniciar su relación actual, solo el 12,1 por ciento de este grupo muestra una resiliencia alta y su media es de 94.40 puntos. El resto responden a una variada situación (casadas, separadas y otra casuística de la que se deriva que ha tenido una relación estable anteriormente), presentan un mayor nivel de resiliencia (el 39,3 por ciento alcanza el nivel alto) y su media es de 100.89 puntos. Tanto los datos porcentuales como la media presentan relevancia estadística $\left(p_{1}<0,01\right.$ y $\left.p_{2}<0,01\right)$. El análisis de la seg- mentación, según las variables establecidas, refleja situaciones similares y, salvo para el grupo de varones de origen extranjero, siempre es menor la puntuación media y el porcentaje de personas con alto nivel de resiliencia en el grupo de las que estaban solteras. Así, en el colectivo nacional, se aprecia una diferencia significativa $\left(p_{1}<0,05\right.$ y $\left.p_{2}<0,05\right)$ de más de siete puntos entre solteros/as tanto en la resiliencia media (93.52 y 101.00 puntos, respectivamente) como en el porcentaje de sujetos con alta resiliencia $(5,48$ por ciento y 27,27 por ciento). Entre las personas extranjeras la diferencia de medias no es relevante (aunque sea superior a los cinco puntos), pero sí lo es $\left(p_{1}<0,05\right)$ el porcentaje de personas con alta resiliencia $(18,42$ por ciento entre solteros/as y 47,06 por ciento para el resto). Igualmente entre las mujeres se observa menor resiliencia en las solteras, tanto en medias (94.55 para las solteras y 102.93 puntos para las demás) como en porcentajes de mujeres con alta resiliencia $(14,86$ por ciento y 53,33 por ciento, respectivamente), siendo elevada la significación estadística $\left(p_{1}<0,01\right.$ y $\left.p_{2}<0,01\right)$.

Podemos afirmar que, aunque la mayoría del colectivo está soltero cuando comienza la relación, es la variable otras situaciones en relación a todos los sujetos la que concentra el 39,3 por ciento de alta resiliencia, mientras que si se analiza según el género, son las mujeres extranjeras las que presentan una mayor resiliencia que las solteras en un 57,1 por ciento de los casos. Puede resultar también llamativo en los supuestos de mujeres extranjeras que las solteras también concentren la tasa más alta con un 23,3 por ciento, muy lejos de los valores de los soltero/a de las otra categorías. Haber mantenido relaciones anteriormente, aunque hayan fracasado o desaparecido, parece estar relacionado con tener mayor resiliencia.

\subsection{La resiliencia, la nacionalidad y el país de origen}

Si se analiza el país de nacimiento de la persona extranjera de la pareja, una vez agrupados en cuatro categorías (Sudamérica, Europa, África y España), se observan bastantes diferencias en la puntuación media de la resiliencia. Así, para la muestra completa, la media de la resiliencia cuando la procedencia es Sud- 
américa o España está por encima de los 98 puntos, dato que está en torno a los 93 puntos si procede de Europa o África. Una situación casi idéntica se observa si se analiza la resiliencia respecto a la nacionalidad del miembro extranjero de la pareja, siendo mayor si es sudamericana (98.21 puntos) o española (97.74). Finalmente, la media de la resiliencia es mayor entre las personas encuestadas si el miembro extranjero tiene la doble nacionalidad -98.53 puntos - que si no tiene dicha doble nacionalidad - 94.82-, diferencia que se observa, especialmente, entre las personas entrevistadas de origen nacional y los hombres.

\section{Discusión y conclusiones}

Las personas que han participado en nuestra encuesta otorgan al sentido de la vida una gran importancia (4.48 puntos de media sobre 5), siendo la variable más valorada. Este hecho se recoge también en la mayoría de las investigaciones sobre resiliencia como uno de los más importantes. Ocurre así en el estudio realizado por Jaramillo et al. (2005) con mujeres maltratadas, que detectaron una correlación positiva entre resiliencia y espiritualidad y establecieron que estos factores contribuyen al autocuidado y la adaptación exitosa. En la misma línea, Cyrulnik (2008) nos plantea que, ante los traumas, un elemento protector muy importante es dotar de significado a lo acontecido. Walhs $(1998,2004)$ considera que el sistema de creencias que poseen las familias es la clave de la resiliencia. Dentro del mismo se incluye dotar de sentido a la adversidad, tener una perspectiva positiva de las dificultades y compartir un sentido de trascendencia y de espiritualidad. Frankl (2003) centra sus investigaciones en este último aspecto y nos indica que hay que encontrarle sentido a la vida, que la búsqueda de la razón de ser de las cosas es la fuerza fundamental que mueve a los seres humanos. Explica que, cuando encontramos un significado a nuestra existencia, nos tranquilizamos, nos sentimos más seguros y fortalecemos nuestra motivación para soportar el dolor y luchar por vencer la adversidad.

Otros aspectos con una puntuación media alta son: mantener interés por nuestras cosas (4.38 puntos) y sentirse orgulloso de los logros alcanzados (4.33 puntos). Ambos permiten que los seres humanos adquiramos conoci- mientos de cómo somos y cómo configuramos una visión realista de nuestros talentos, recursos y defectos. Esta función también nos ayuda a entender las causas de nuestros pensamientos, emociones y actos, así como a encontrar explicaciones a los acontecimientos que vivimos (Vanistendael, 1996; Fernández, 2004; Cyrulnik, 2008; Fiorentino, 2008; Rojas, 2010). Supone ser capaces de examinarnos internamente, plantearnos cuestiones difíciles y darnos respuestas honestas. El desarrollo de esta capacidad ejecutiva se verá favorecida por el esfuerzo, el entrenamiento, con una actitud abierta, y la toma de conciencia de los beneficios que tiene que nos examinemos y nos conozcamos. Dentro de esta función se sitúa también el autocontrol, entendido como «la aptitud para frenar conscientemente los ímpetus, para frenar y retrasar voluntariamente la gratificación inmediata, con el fin de conseguir un objetivo superior» (Rojas, 2010, p. 72). Esta capacidad se desarrolla desde la infancia y afecta a todos los aspectos de nuestra vida, llegando a constituir una de las piedras angulares de los individuos resilientes. Vanistendael (1996) y Goleman (2004) destacan la autogestión como un elemento clave de la inteligencia emocional y el liderazgo efectivo. Asimismo, consideramos que la autoestima también es un elemento fundamental que se ha de relacionar con estos ítems, porque proporciona la confianza en uno mismo, necesaria para enfrentarse a los problemas y sentir orgullo por los logros alcanzados (Werner y Smith, 1993; Brooks y Goldstein, 2004).

Considerarse una persona con sentido del humor (4.33 puntos de media) pone de manifiesto la importancia de la función adaptativa de las emociones positivas para solucionar cuestiones relativas al desarrollo, el crecimiento personal y la conexión social. Mejoran nuestra forma de pensar, nuestra salud y nuestras capacidades de afrontamiento de la adversidad. La perspectiva positiva de las cosas no sólo nutre directamente la capacidad para encajar desgracias y la motivación para superarlas, sino que además vigoriza y fortifica otros ingredientes de la resiliencia, como las conexiones afectivas, las funciones ejecutivas, la autoestima, la búsqueda de un significado positivo de la vida y la identificación de motivos relevantes que de significado y motivos a la vida. En palabras de Vanisten- 
dael: «tal vez no podamos librarnos del sufrimiento pero no acabará con nosotros») (1996, p.26). Todo esto indica que la auténtica gracia puede ser algo más útil que un simple mecanismo de escape, ya que no se pretende evadirse o ignorar la realidad sino hacer de ella algo más llevadero, soportable y positivo. En todas las culturas existe alguna forma de sentido del humor, que es «un elemento de gestión con efectos liberadores» (Cyrulnik, 2008, p. 97).

En nuestra investigación ser mujer y extranjera resulta una combinación favorecedora de la resiliencia. Sin embargo, no existe un acuerdo absoluto, en la bibliografía consultada, respecto a la influencia del género. Nos encontramos investigaciones - las de Lundman, et al. (2007), Saavedra y Villalta (2008) y Rodríguez et. al. (2009) — , que ponen de manifiesto que el género por sí mismo no es determinante de resiliencia. Y, por otro lado, hay investigaciones que muestran diferencias entre las respuestas de hombres y mujeres - Prado y Del Aguila, (2003) y Walsh (2004) — que afirman que las mujeres superan la adversidad en un mayor porcentaje que los varones. Esto podría explicarse, según las autoras, por el tipo de socialización, ya que a las niñas se les enseña a ser más amables, sociables y a desarrollar estrategias más abiertas y tendentes a buscar apoyos en el entorno mientras que a los varones, a quienes se les enseña a confiar sobre todo en sí mismos y presentar imágenes más duras que se alejan de las estrategias favorecedoras del desarrollo de la resiliencia. Grotberg (2008) muestra que la resolución de conflictos entre niños y niñas es diferente, tendiendo las niñas a hacer uso de habilidades interpersonales y fortalezas internas en tanto los chicos tienden a ser más pragmáticos. Rutter $(1985,1987)$ encontró grandes diferencias en la manera de interaccionar en el contexto: los chicos expuestos a conflictos familiares desarrollan más fácilmente perturbaciones emocionales y de conducta que las chicas.

Finalmente, en cuanto a la edad, las investigaciones de Warnild y Young (1993) y Lund- man et al. (2007) encontraron que es una variable de vital importancia en la resiliencia. Así sucede en nuestra investigación, donde correlaciona positivamente con la misma, que se desarrolla a lo largo de la vida, aumentando con el paso del tiempo. Tusai y Dyer (2004) estudiaron este desarrollo en las diferentes etapas evolutivas del ser humano, sosteniendo que puede estar condicionado e influenciado por factores personales, familiares y de apoyo de la comunidad.

Como reflexión final de nuestro trabajo, queremos poner de manifiesto que, si bien el conjunto de personas entrevistadas tienen un nivel de resiliencia medio moderado, éste aumenta cuando se combinan poseer origen extranjero y ser mujer. Igualmente, es significativo que las mujeres extranjeras con más resiliencia, desde el punto de vista del estado civil, son aquellas que no están solteras y que han vivido otras relaciones que resultaron un fracaso. Aunque tienen pocos niños, nosotros consideramos que abordar su crianza en solitario es un factor que aumenta la resiliencia de estas mujeres. No obstante, como ya hemos señalado, la literatura existente no revela con contundencia que el género sea un factor determinante para poseer altos niveles de resiliencia. Por esta razón, creemos de interés poder abordar investigaciones futuras sobre la relación de género y resiliencia en la inmigración

En el grupo analizado, los ítems que alcanzan mayor puntuación se sitúan en el ámbito de la competencia personal y la autoeficacia. Que la vida tenga sentido, mantener interés por las cosas que nos son importantes, ser alguien en quien los otros puedan confiar y el sentido del humor se configuran como los aspectos más valorados. Por ello creemos necesario profundizar en la relación existente entre la resiliencia y los sistemas de creencias, desde un doble dimensión: personal (de cada miembro de la pareja) y compartido (el sistema de creencias familiar), así como los mecanismos y procesos claves desde donde se construyen.

\section{Referencias bibliográficas}

Alaminos, A. (2008). Matrimonios mixtos europeos. Un modelo empírico. Revista OBETS, 2, 131-149.

Albert, M. C. y Massanet, E. (2008). Los matrimonios mixtos en España ¿espacios de construcción intercultural? Revista OBETS, 1, 45-71. 
Barranco, C. (2009). Trabajo social, calidad de vida y estrategias resilientes. Portularia, 9, 133-145.

Borobio, D. (Coord.) (2003). Familia e Interculturalidad. Salamanca: Universidad Pontificia de Salamanca.

Brooks, R. y Goldstein, S. (2004). El poder de la resiliencia. Barcelona: Paidós.

Cyrulnik, B. (2008). Los patitos feos. La resiliencia: una infancia infeliz no determina la vida. (9a ed). Barcelona: Gedisa.

Esteve, A. y Cortina, C. (2009). Cohabitación y endogamia entre la población extranjera en España: pautas diferenciales por origen. Albert Esteve et al., Parejas binacionales en la sociedad avanzada: realidades y tendencias de la hibridación transcultural (pp. 5-29). Sevilla: Fundación Centro de Estudios Andaluces.

Fernández, D. (2004). Resiliencia y adolescencia. En Fernández D'Adams (coord.), Resiliencia, ética y prevención. Buenos Aires: Gabas.

Fiorentino, M. T. (2008). La construcción de la resiliencia en el mejoramiento de la calidad de vida y la salud. Suma psicológica, 15, 95-104.

Frankl, V. (2003). Psicoterapia y existencialismo. Barcelona: Herder.

Garmezy, N. (1991). Resiliency and vulnerability to adverse developmental autcomes associated with poverty. American Behavioral Scienctist, 34, 416-430. Disponible en: http://es.youscribe.com/catalogue/tous/otros/resiliencia-psicologica-y-dolor-cronico-psychological-resilienceand-2007824.http://dx.doi.org/10.1177/0002764291034004003

Giménez, C. (2003). Familias e inmigración. Su integración en la sociedad receptora. En D. Borobio (coord.), Familia e Interculturalidad. (pp. 129-196). Salamanca: Universidad Pontificia de Salamanca.

Grotberg, E. (2008). El poder de la resiliencia: adolescentes contra la violencia. En A. Melillo, E. Suárez y D. Rodríguez (comps.), Resiliencia y subjetividad. Los ciclos de la vida. (pp. 155172). Buenos Aires: Paidós.

Goleman, D. (2004). Inteligencia emocional. Barcelona: Kairós.

Heilemann, M. V., Lee, K. y Kury, F. S. (2003). Psychometric Properties of Spanish Version of the Resilience Scale. Journal of Nursing Measurement, 1, 61-72. Disponible en: http://dx.doi.org/10.1891/jnum.11.1.61.52067

Jaramillo, D. E., Ospina-Muñoz, D. E, Cabarcas, G. y Humphreys, J. (2005). Resiliencia, Espiritualidad, Aflición y Tácticas de Resolución de conflictos en Mujeres Maltratadas. Revista Salud Pública, 7, 291-282. Disponible en: http://dx.doi.org/10.1590/S0124-00642005000300004

Kalmijn, M. (1998). Intemarriage and homogamy: Causes, Patterns and Trends. Anual Review, 56, 786-800.

Kotliarenco, M. A. y Dueñas, V. (1994). Vulnerabilidad versus resiliente: Una propuesta de acción educativa. Revista Derecho a la Infancia, 9, 2-22.

Kotliarenco, M. A., Cáceres, I. y Álvarez, C. (1996). La pobreza desde la mirada de la resiliencia. En Construyendo en adversidad (pp. 24-33). Santiago: CEANIM.

Kotliarenco, M. A., Cáceres, I. y Fonteccilla, M. (1997). Estado del arte de la resiliencia. Washington. D.C.: Oficina Panamericana de la Salud.

Lundman, B., Strandberg, G., Eisemman, M., Gustafson, Y. y Brulin, C. (2007). Psychometric Properties of the Version of de Resilience Scale. Scandinavian Journal of Caring Sciences, 21(2), 229-237. Disponible en: http://dx.doi.org/10.1111/j.1471-6712.2007.00461.x

Luttar, S. S. (1991). Vulnerabiliti and resilience: A study of high- risk adolescent. Child Development 62(3), 600-616. Disponible en: http://dx.doi.org/10.2307/1131134

Masten, A. S., y Coastworth, J. D. (1998). The developent of competente in favorable and unfavorable environments. American Psychologist, 53, 205-220. Disponible en: http://dx.doi.org/10.1037/0003066X.53.2.205

Muñoz-Silva, A. (2012). El estudio de la resiliencia desde la perspectiva evolutiva y su aportación a la comprensión del riesgo y la protección en la intervención social. Portularia 12(1), 9-16. Disponible en: http://dx.doi.org/10.5218/prts.2012.0029 
Novella, A. (2002). Incremento de la resiliencia luego de la aplicación de un programa de psicoterapia breve en madres adolescentes. (Tesis doctoral inédita). Universidad Nacional Mayor de San Marcos, Lima. Perú.

Palma García, M. O. (2010). La resiliencia en Trabajo Social. (Tesis doctoral inédita). Universidad de Málaga.

Pesce, R. P., Assis, S. G., Avanci, J. Q., Santos, N. C., Malaquías, J. V. y Carvalhaes, R. (2005). Adaptacao transcultural, confiabilidade e validade da escala de resiliencia. Saude Pública, 21(2), 436-448. Disponible en: http://dx.doi.org/10.1590/S0102-311X2005000200010

Prado, R., y Del Águila, M. (2003). Diferencia en la resiliencia según género y nivel socioeconómico en adolescentes. Persona, 6, 179-196.

Rodríguez, M., Pereyra, M., Gil, E. Jofre, M. De Bortoli, M. y Labiano, L. (2009). Propiedades psicométricas de la escala de resiliencia Versión Argentina. Laboratorio de evaluación Psicológica y Educativa. Evaluar, 9, 72-82.

Rodríguez García, D. (2004). Inmigración y mestizaje hoy. Formación de matrimonios mixtos y familias trasnacionales de inmigrantes en Cataluña. Migraciones 16, 77-120.

Rodríguez Marcos, M. E. (2006). Familias interculturales,. La construcción de la interculturalidad de lo microsocial a lo macrosocial. Salamanca: Publicaciones Universidad Pontificia de Salamanca.

Rojas, L. (2010). Superar la adversidad. El poder de la resiliencia. Madrid: Espasa.

Rutter, M. (1985). Resiliene in the face of adversiy. Protective factors and resistence to psychiatrics disorder. Br. J. Psychiatry, 147, 598-611. Disponible en: http://dx.doi.org/10.1192/ bjp.147.6.598

Rutter, M. (1987). Psychosocial resilience and protective mechanismisms. American Journal Orthopsychiatry 57(3), 316-319. Disponible en: http://dx.doi.org/10.1111/j.1939-0025.1987.tb03541.x

Saavedra, E. y Villalta, M. (2008). Medición de las características resilientes, un estudio comparativo entre personas de 15 a 65 años. Liberabit, 14, 31-40.

Tognetti-Bordogna, M. (1996). Legami familiari e inmigrazione: i matrimoni misti, Torino: L`Harmattan Italia.

Tusai, K. y Dyer, J. (2004). Resilience: a historical review of the construct. Holist Nurs Prat, 18, 3-8.

Vanistendael, S. (1996). Cómo crecer superando los percances. Resiliencia: capitalizar las fuerzas del individuo. Ginebra: Internacional Catholic Child Bureau.

Villalba, C. (2003). El concepto de resiliencia individual y familiar. Aplicaciones para la intervención social. Intervención psicosocial, 12, 283-299.

Villalba, C. (2004). La perspectiva ecológica en trabajo social con infancia, adolescentes y familia. Portularia, 4, 287-298.

Villalba, C. (2006). El enfoque de resiliencia en trabajo social. Acciones e investigaciones sociales, Extra 1, 446.

Walhs. F. (1998). Family resilience: a concept an its applications: The concept of familiy resilience, crisis and chanllenge. Family process Journal, 35(3), 261-287.

Walhs. F. (2004). Resiliencia Familiar. Estrategias para su afrontamiento. Buenos Aires: Amorrortu.

Wagnild G. y Young HM (1993). Development and psychometric evaluation of the Resilience Scale. Journal of Nursing Measurement, 1, 65-178.

Werner, E. y Smith, R. (1992). Vulnerable but invincible: A longitudinal study of resilient children and young. Nueva York: McGraw-Hill.

Werner, E. y Smith, R. (1993). Overcoming the odds: high risk children from birth to adulthood. Ithaca: Cornell University Press. 